\title{
Completing the Mechanical Energy Pathways in Turbulent Rayleigh-Bénard Convection
}

\author{
Bishakhdatta Gayen, ${ }^{*}$ Graham O. Hughes, and Ross W. Griffiths \\ Research School of Earth Sciences, Australian National University, Canberra, Australian Capital Territory 0200, Australia
} (Received 29 April 2013; published 17 September 2013)

\begin{abstract}
A new, more complete view of the mechanical energy budget for Rayleigh-Bénard convection is developed and examined using three-dimensional numerical simulations at large Rayleigh numbers and Prandtl number of 1 . The driving role of available potential energy is highlighted. The relative magnitudes of different energy conversions or pathways change significantly over the range of Rayleigh numbers $\mathrm{Ra} \sim 10^{7}-10^{13}$. At $\mathrm{Ra}<10^{7}$ small-scale turbulent motions are energized directly from available potential energy via turbulent buoyancy flux and kinetic energy is dissipated at comparable rates by both the largeand small-scale motions. In contrast, at $\mathrm{Ra} \geq 10^{10}$ most of the available potential energy goes into kinetic energy of the large-scale flow, which undergoes shear instabilities that sustain small-scale turbulence. The irreversible mixing is largely confined to the unstable boundary layer, its rate exactly equal to the generation of available potential energy by the boundary fluxes, and mixing efficiency is $50 \%$.
\end{abstract}

DOI: 10.1103/PhysRevLett.111.124301

PACS numbers: 44.25.+f, 47.27.E-, 47.55.pb

Introduction.-Flow in a horizontal layer of fluid heated uniformly from below and cooled from above-RayleighBénard convection (RBC) - is an idealized problem from which much can be learned about the nature of convective flow and heat transfer. Recent attention has focused on the relative importance of the boundary layer and interior behavior, including flow structures, at very large Rayleigh numbers [1-6]. The salient features of the flow can be usefully interpreted in terms of the energy budget, and previous work has considered the kinetic and thermal energy $[2,3,7,8]$. However, the existing framework is incomplete and provides limited insight into the exchange of mechanical energy among different forms or between fluid motions of different scales. In particular, the nature of the turbulent energy cascade in RBC is still not fully understood [3] and improved models are needed.

Convection is caused by the generation of mechanical energy in the form of available potential energy (APE) by boundary buoyancy fluxes. APE is a subset of the total gravitational potential energy (GPE) and is the form essential for motions [9-11]. A complete mechanical energy framework has been recently developed for the case of horizontal convection forced by differential heating at one horizontal boundary $[12,13]$ and recently extended to $\mathrm{RBC}$ [14]. In RBC there is a release of APE from thermal boundary layers adjacent to the top and bottom boundaries, driving flow at various scales, while complex feedbacks further distribute the potential and kinetic energy among the different scales or, through mixing, to the background potential energy. The role of APE in RBC was recognized in quantifying the overall rate of irreversible mixing [14]. Here we show that an account of GPE, its partition into available and background forms, and its links to kinetic energy at various scales are essential in a full understanding of the turbulent energy cascade and the relative roles of the boundary layer and the interior.
Problem setup.-The computational domain consisted of a rectangular box of height $H$, with bottom and top boundaries at $z=-0.5 H, 0.5 H$, and horizontal dimensions $L \times L$. The aspect ratio of the domain for most solutions was $A=$ $L / H=3$, as we are most interested in wide layers, without sidewall effects. Periodicity was imposed in the horizontal $(x, y)$ directions on velocity, pressure, and temperature. Constant uniform temperatures $T_{H}$ and $T_{C}$ were applied at the bottom and top boundaries of the domain, respectively, along with no-slip velocity boundary conditions. The simulations covered the Rayleigh number range $6 \times 10^{6} \leq$ $\mathrm{Ra} \leq 6 \times 10^{12}$ with a fixed Prandtl number $\operatorname{Pr}=1$. Direct numerical simulations (DNS) used a $512^{3}$ grid, stretched in the $z$ direction, up to moderately large Rayleigh numbers Ra $\leq 6 \times 10^{8}$. Large eddy simulations (LES) were used for $6 \times 10^{6} \leq \mathrm{Ra} \leq 6 \times 10^{12}$ (with grids up to $1024^{3}$ ). Additional DNS at Ra $=6 \times 10^{8}$ were carried out for aspect ratios of $A=1.5$ and $A=6$.

The three-dimensional, unsteady Navier-Stokes equations under the Boussinesq approximation were solved. Horizontal derivatives were treated with a pseudospectral method and the vertical derivatives were computed with second-order finite differences. A third-order Runge-KuttaWray method was used for time stepping, and viscous terms were treated implicitly with the Crank-Nicolson method. All DNS solutions satisfied standard convergence criteria [15] (with grid spacing $l \leq \pi \eta, \eta$ the Kolmogorov scale). The subgridscale model in the LES was based on a dynamic eddy viscosity and eddy diffusivity (with $l \leq 10 \eta$ ), as previously shown to be accurate for convection [16-19] (the detailed algorithms are found in [20,21]). The LES solutions were also validated against the DNS solutions at $\mathrm{Ra} \leq 6 \times 10^{8}$ and showed no significant differences (in heat transport, volume integrals and vertical profiles of energy conversion terms, and dissipation spectra). Exact closure of the energy budget (based on independent 
(a)

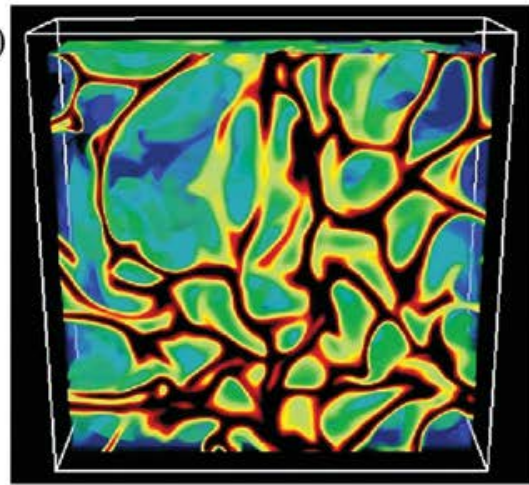

(b)

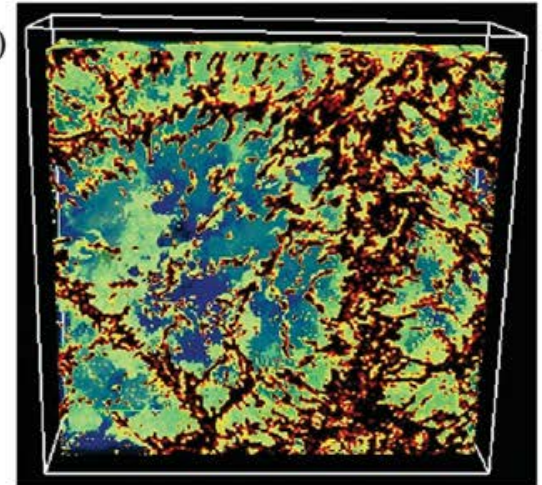

(c)

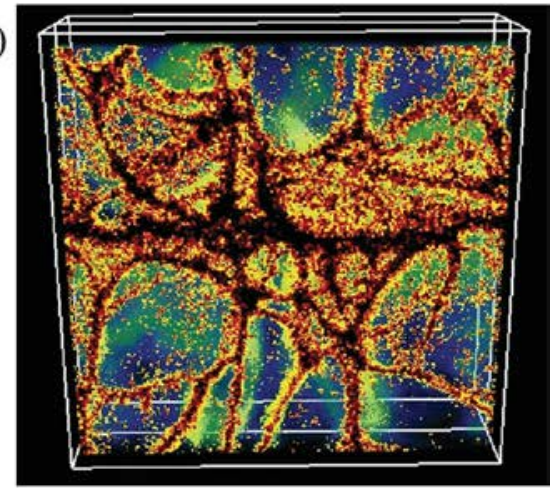

FIG. 1 (color). Visualization of the thermal structures by color contours of the 3D temperature field, normalized as $\left(T-T_{C}\right) / \Delta T$, inside a subdomain $0.3 \delta_{95}<z<H / 2$ viewed from the bottom, at (a) $\mathrm{Ra}=6 \times 10^{6}$, (b) $\mathrm{Ra}=6 \times 10^{8}$, and (c) $\mathrm{Ra}=6 \times 10^{12}\left(\delta_{95}\right.$ is the thickness of the thermal boundary layer incorporating $95 \%$ of $\Delta T / 2$ ). Dark red indicates the warmest fluid.

calculations of each term below; see [13]) was also obtained in both DNS and LES solutions.

Results.-The change in flow structure over the range of $\mathrm{Ra}$ (Fig. 1) is remarkable. At $\mathrm{Ra} \sim 10^{7}$ the convection is more or less homogeneous and forms cell-like structures. Smaller scales appear with increasing Ra [Fig. 1(b)], and become increasingly organized at still larger Ra [Fig. 1(c)] as much larger scales of coherent motion emerge (e.g., Ref. [22]). The Nusselt number ( $\mathrm{Nu}$ ) scaling with Rayleigh number (Fig. 2) agrees well with previous experimental data $[23,24]$ and with DNS results for RBC in a closed cylindrical geometry of aspect ratio $A \sim 0.25-0.5$ [4]. The agreement includes a break in slope at $\mathrm{Ra} \sim 10^{8}$. The present LES runs accurately capture heat transfer and reproduce the DNS results at $\mathrm{Ra} \leq 6 \times 10^{9}$. The overall heat transport is also insensitive to aspect ratio. At the highest Ra considered here, there is no sign of a transition to the ultimate regime of Kraichnan [25,26]. The observed

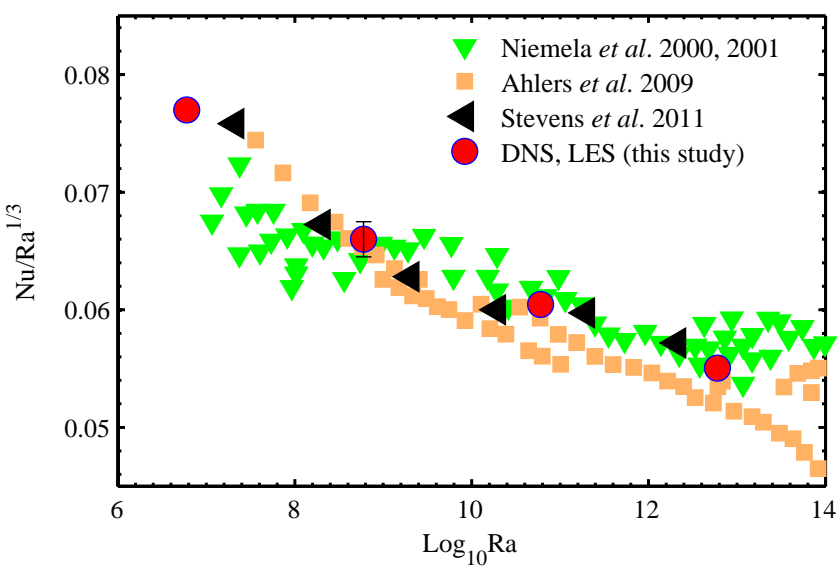

FIG. 2 (color online). Values of $\mathrm{Nu} / \mathrm{Ra}^{1 / 3}$ obtained here (red) as a function of $\mathrm{Ra}$, and comparison with previous works. Results at aspect ratios of $A=1.5,3,6$ and $\mathrm{Ra}=6 \times 10^{8}$ all lie within the range of the bar shown at that $\mathrm{Ra}$. The present DNS and LES at $\mathrm{Ra}<10^{9}$ are indistinguishable from each other.
$\mathrm{Nu}(\mathrm{Ra})$ scaling never exceeds the $1 / 3$ power law, but a change of slope occurs around $\mathrm{Ra} \sim 10^{9}$, consistent with previous DNS [27] and experimental results [23,24]. The mechanical energy budget developed here is useful in exploring the reason for this transition. Other experimental results have indicated departure from the trend in Fig. 2 at $\mathrm{Ra}>10^{11}[28]$ or $\mathrm{Ra}>10^{12}-10^{13}$ [29]. Our results are not significantly different from the latter of these.

The proposed energetics framework for $\mathrm{RBC}$ in a Boussinesq fluid is shown schematically in Fig. 3. This framework is broadly similar to that proposed for the global ocean circulation [12] and recently implemented for horizontal convection [13], but it is modified here for the RBC case. Mechanical energy includes both kinetic energy (KE) $E_{k}=\rho_{0} / 2 \int u_{i} u_{i} d V$ and GPE

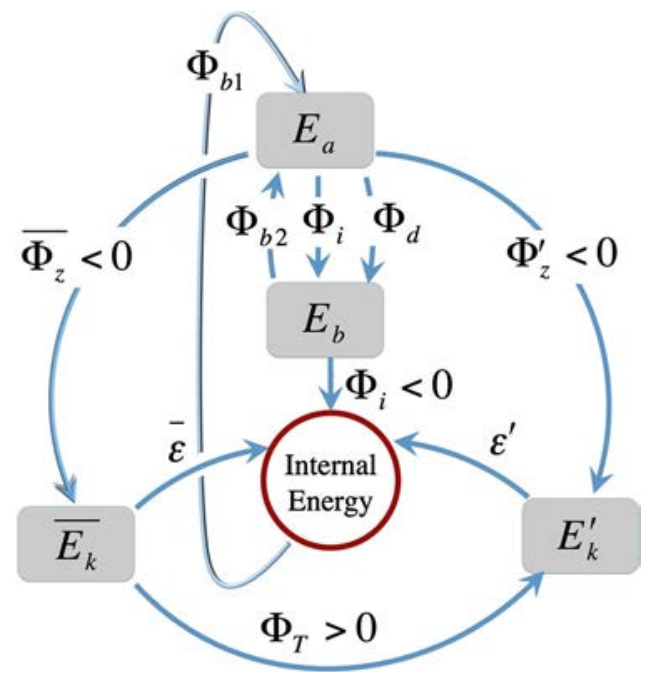

FIG. 3 (color online). Schematic diagram of the forms and conversions of mechanical energy in RBC of a Boussinesq fluid (adapted from [12]; the $\Phi_{b 1}$ pathway and direction of all conversions are modified for RBC as in [14] with decomposition of the KE loop into mean and fluctuating contributions). See text for details. 
$E_{p}=g \int z \rho d V$, and energy transfer between these forms is facilitated by buoyancy fluxes, $\Phi_{z}=g \int \rho w d V$. Here, $u_{i}$ is the velocity component in the $i$ th direction, $\rho$ is the density, $\rho_{0}$ is the reference density, and $g$ is the gravitational acceleration. The volume integrals are taken over the full depth of the fluid and horizontal distances suitably large compared to the significant scales of motion.

The potential energy is further decomposed into background potential energy (BPE) $E_{b}=g \int z_{*} \rho d V$ (which cannot drive motion) and available potential energy, $E_{a}=E_{p}-E_{b}[9,10]$. The BPE of a volume of fluid corresponds to $E_{p}$ for a state of no motion, or gravitational equilibrium, in which fluid parcels have been adiabatically rearranged to new vertical positions $z_{*}=z_{*}(\rho)$.

Detailed derivations of the energy conversion rates in Fig. $3[12,14]$ show that exchange between APE and BPE is via irreversible mixing $\Phi_{d}=-g \kappa \int\left(d z_{*} / d \rho\right) \times$ $\left(\partial \rho / \partial x_{i}\right)^{2} d V$, a lowering of the center of mass by molecular diffusion down the background gradient $\Phi_{i}=$ $-g \kappa L^{2}\left(\langle\rho\rangle_{H / 2}-\langle\rho\rangle_{-H / 2}\right)$, and by buoyancy input through the boundaries $\Phi_{b 2}=g \kappa \oint z_{*}\left(\partial \rho / \partial x_{i}\right) n_{i} d S$, where $\kappa$ is the molecular diffusivity of heat (and buoyancy), \langle\rangle$_{z}$ denotes a horizontal area average at height $z$, and $S$ is the surface (with normal $n_{i}$ ) bounding the volume. For $\operatorname{RBC} \Phi_{b 2}$ represents the rate of energy input required to maintain a density field away from an adiabatically relaxed state, and can be expressed as $\Phi_{b 2}=\rho_{0} \kappa g \alpha \Delta T N u L^{2}$, where $\alpha$ is the expansion coefficient [14]. Kinetic energy is dissipated by viscosity at the rate $\varepsilon=\rho_{0} \nu \int\left(\partial u_{i} / \partial x_{j}\right)^{2} d V$, which can be expressed in the form [8] $\varepsilon=\rho_{0} \nu^{3}$ $(\mathrm{Nu}-1) \operatorname{RaPr}^{-2} L^{2} / H^{3}$. Mechanical energy is converted between internal and potential energy by both $\Phi_{i}$ and the net buoyancy input (i.e., the rate of change of available potential energy by net heating or cooling) at the boundaries $\Phi_{b 1}=g \kappa \oint z\left(\partial \rho / \partial x_{i}\right) n_{i} d S$. Note that while the mechanical energy and its transfer to and from internal energy involves heat fluxes and dissipation, it can be considered separately from the heat budget within the Boussinesq approximation. A discussion can be found in Ref. [30]. We have already shown [14] that inclusion of the two potential energy forms in the previous kinetic energy framework for $\mathrm{RBC}$ is required to theoretically predict the rate of irreversible mixing. However, the energy pathways between the different scales of motion could not be predicted without numerical simulation.

Progress is made here by decomposing the kinetic energy of the fluid into mean, $\bar{E}_{k}=\rho_{0} / 2 \int \bar{u}_{i} \bar{u}_{i} d V$, and turbulent, $E_{k}^{\prime}=\rho_{0} / 2 \int \overline{u_{i}^{\prime} u_{i}^{\prime}} d V$, contributions. The mean (overbar) velocity and density components are spatially dependent and obtained by time averaging data over three turnover times, $\tau_{0}(=\sqrt{H / g \alpha \Delta T})$. Fluctuating quantities (primed) during the averaging period are calculated as deviations from the mean. The results are insensitive to averaging times in the range (2-6) $\tau_{0}$. Small-scale turbulent motions must be sustained by extracting kinetic energy from the mean flow [the shear production $\Phi_{T}=-\rho_{0} \int \overline{u_{i}^{\prime} u_{j}^{\prime}}\left(\partial \bar{u}_{i} / \partial x_{j}\right) d V$ ] or from APE (the turbulent buoyancy flux $\Phi_{z}^{\prime}=g \int \overline{\rho^{\prime} w^{\prime}} d V$, Fig. 3). The large-scale motions, on the other hand, are maintained only by the mean buoyancy flux $\bar{\Phi}_{z}=$ $g \int \bar{\rho} \bar{w} d V$. Note that we avoid mean quantities based on horizontal averaging (e.g., Refs. [7,31]), which would disguise contributions to $\bar{\Phi}_{z}$ associated with relatively long-lived large-scale structures (that are prevalent at the higher Ra).

The energy conversion rates found from both DNS and LES confirm for all Ra that the rate of loss of APE (and increase of BPE) due to irreversible mixing is exactly equal to the rate of generation of APE from BPE by boundary forcing $\left(\Phi_{d}=\Phi_{b 2}\right.$, as theoretically predicted $[12,30]$ and shown to be the case in DNS of horizontal convection [13]). For $\operatorname{RBC} \Phi_{i}$ is negative but negligible $\left[\Phi_{i} / \varepsilon=\right.$ $1 /(1-\mathrm{Nu})$, Ref. [12]]. The remaining key conversion rates are compared in Fig. 4.

At the smallest Rayleigh number considered $\left(6 \times 10^{6}\right)$, energy is transferred to both mean and fluctuating kinetic energy via buoyancy flux (the left and right pathways in Fig. 3) at comparable rates, although the fluctuating contribution is the smaller. Further generation of small-scale flows by shear production $\Phi_{T}$ is small. Thus small-scale convection is the primary source of turbulent motions. Viscous dissipation of the mean (large-scale) motions $\bar{\varepsilon}$ and of the small-scale motions $\varepsilon^{\prime}$ occur at similar rates.

At larger Rayleigh numbers the large-scale circulation (or "wind") develops [compare Figs. 1(b) and 1(c)], as previously described [24,27]. Accompanying this change in the flow is a substantial change in the energy pathways. More energy is pumped into kinetic energy through the mean buoyancy flux (left-hand pathway in Fig. 3), as a result of small-scale convective plumes being swept along the boundary layer and merging into larger scales

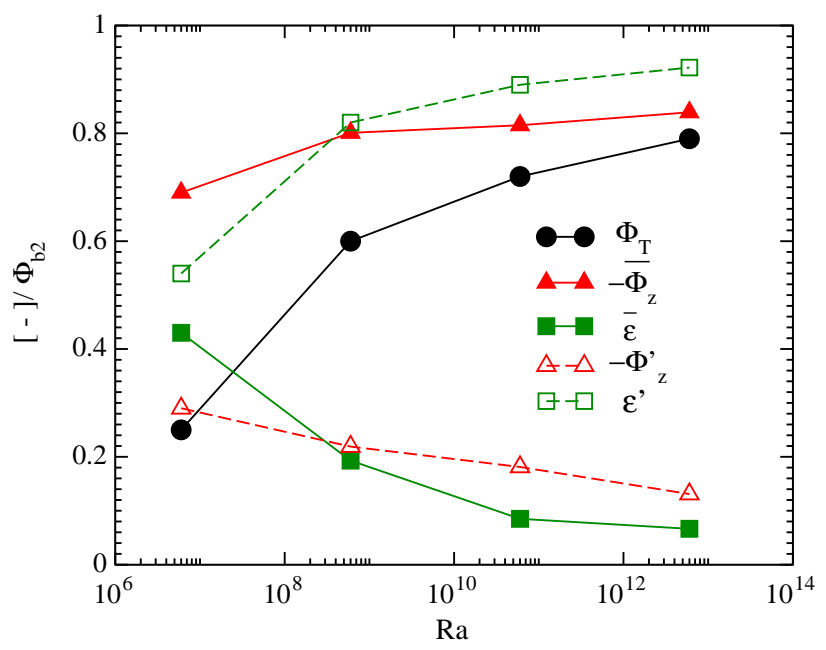

FIG. 4 (color online). Time-averaged, domain-integrated conversion rates for kinetic energy as functions of Ra. Conversions are normalized by the rate of APE generation by boundary buoyancy fluxes $\left(\Phi_{b 2}\right)$. 
$[2,22,32]$. The changes in $\bar{\Phi}_{z}$ and $\Phi_{z}^{\prime}$ are gradual with Ra, but the ratio $\bar{\Phi}_{z} / \Phi_{z}^{\prime}$ increases rapidly at $\mathrm{Ra}>10^{11}$. Viscous dissipation of the large-scale structures is inefficient and its relative role decreases with increasing Ra. The results also show that the wind velocity associated with the large-scale structure is larger at the larger $\mathrm{Ra}$ and the velocity boundary layer thickness is significantly smaller, resulting in enhancement of the boundary shear $[2,33]$ and production of small-scale motions (the bottom arrow in Fig. 3). At $\mathrm{Ra} \sim 10^{13}$ almost $80 \%$ of the turbulence is produced by shear; only $20 \%$ comes directly from APE via the turbulent buoyancy flux (the right-hand pathway) associated with small-scale convective plumes. The associated turbulent kinetic energy is then dissipated $\left(\varepsilon^{\prime}\right.$ in Fig. 3). Thus, convection at the higher Rayleigh numbers predominantly involves energy conversion from APE to mean $\mathrm{KE}$, from mean $\mathrm{KE}$ to turbulence $\mathrm{KE}$ via shear instability, and dissipation of KE.

The relative contributions of the different energy conversions in the boundary layer and in the bulk of the fluid are shown in vertical profiles (Fig. 5). A comparison of the corresponding profiles at $\mathrm{Ra}=6 \times 10^{8}$ shows no significant differences between DNS and LES, along with behavior intermediate to those in Fig. 5. Although the peak turbulent dissipation occurs at the bottom and top boundaries for both cases shown, the role of turbulent dissipation spreads well into the interior, and overall is significantly larger, at the larger $\mathrm{Ra}$, a result consistent with the predictions of the Grossmann-Lohse model [2,33,34]. Nevertheless, normalized dissipation is little changed

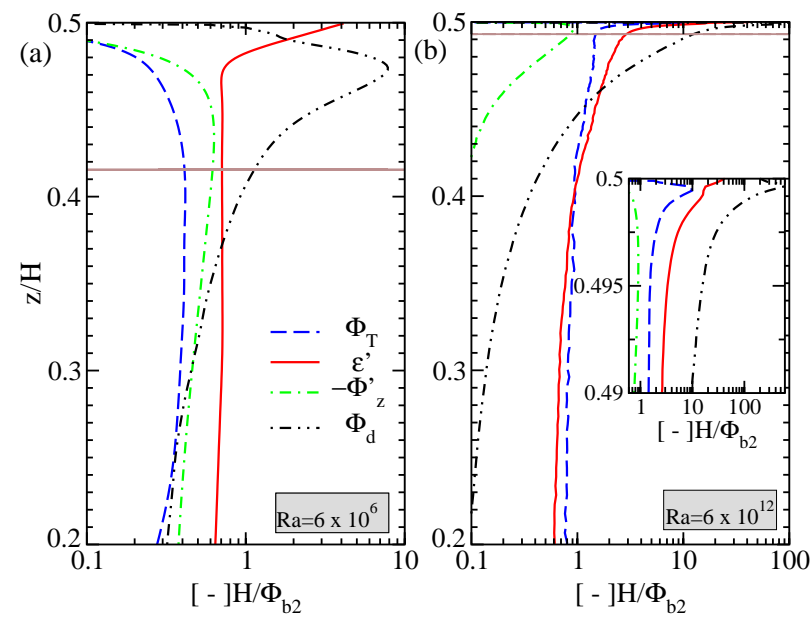

FIG. 5 (color online). (a) Vertical profiles of contributions to turbulence production $\Phi_{T}$, dissipation $\varepsilon^{\prime}$, buoyancy flux $\Phi_{z}^{\prime}$, and irreversible mixing $\Phi_{d}$ at (a) $\mathrm{Ra}=6 \times 10^{6}$ and (b) $\mathrm{Ra}=6 \times 10^{12}$. Contributions are horizontally averaged and normalized by the corresponding domain-averaged value of $\Phi_{b 2} / H$. Plots show the upper $30 \%$ of the domain, horizontal brown line indicates the edge of the upper thermal boundary layer $\delta_{95}$. Inset in (b) shows a zoomed view of profiles in the boundary layer. over the central $70 \%$ of the domain. Furthermore, the irreversible mixing at large $\mathrm{Ra}$ becomes even more strongly concentrated in the boundary layer. In fact, the irreversible mixing rates in the interior (even when volume integrated) are substantially smaller at large $\mathrm{Ra}$ because the thermal gradient in the bulk of the fluid is much weaker. At $\mathrm{Ra} \sim 10^{7}$ the turbulent buoyancy flux, which supplies approximately $50 \%$ of the power for the small-scale motions (Fig. 4), acts throughout the domain with only a weak maximum inside the boundary layer. The source of the other $50 \%$ is turbulent shear production, which has a similar profile to the buoyancy flux. At $\mathrm{Ra} \sim 10^{13}$, however, shear production plays a dominant role in the energy exchange from the large-scale flow to the turbulent motions at all depths, including within the boundary layer where it has a large peak close to the wall. Note that at this $\mathrm{Ra}$ the total contribution of shear production in the bulk of the fluid is much larger than the total in the boundary layer volume and that, in each region, the shear production is large compared with the conversion from APE to KE by the turbulent buoyancy flux. Another important result is that the simulations at $\mathrm{Ra}>10^{10}$ revealed the logarithmic temperature profile typical of non-convecting turbulent boundary layers, as reported by Ref. [5]. These observations support the conclusion that dissipation is dominated by the interior turbulence.

Enhanced boundary shear from the "wind" at the larger $\mathrm{Ra}$ is reflected in the flow structures (see Fig. 1). At $\mathrm{Ra} \sim 10^{7}$ the cell structures are coherent and bounded by lines of flow convergence, which represent the base of ascending sheetlike plumes $[1,19,35]$. The structure changes significantly at larger Ra; the sheet plumes tend to lie perpendicular to the "wind" direction and migrate and merge into a "megaplume." Although there is already a tendency to form megaplumes at $\mathrm{Ra} \sim 10^{9}$, the signature of the large-scale structures and well-organized convergence lines is clear at $\mathrm{Ra} \sim 10^{13}$ [Fig. 1(c)]. Similar observations were made in experiments at $\mathrm{Ra} \sim 10^{11}$ [35]. At these large $\mathrm{Ra}$ there is no longer a homogeneous distribution of small convective plumes inside the boundary layer, reflecting the weak energy transfer from APE to fluctuating KE via the turbulent buoyancy flux (Fig. 4).

Finally, we note that Rayleigh-Bénard convection can be characterized as an efficient mechanism for heat transport. Following extensive work with turbulent stratified flows, the mixing efficiency $\eta$ for convective flow is defined as the proportion of the kinetic energy "lost" to irreversible mixing, compared with the total "loss" due to mixing and viscous dissipation [11-13]: $\eta=\left(\Phi_{d}-\Phi_{i}\right) /\left(\Phi_{d}-\Phi_{i}+\varepsilon\right)$. In the present simulation at the largest Ra this quantity evaluates to $\eta \approx 0.5001$, in good agreement with the theoretical prediction for RBC, $\eta \rightarrow 1 / 2$ at large Ra [14]. A similarly high mixing efficiency has been reported for Rayleigh-Taylor experiments [36] and may be a general characteristic of convective flows. 
Conclusions.-The energy budget and pathways for Rayleigh-Bénard convection have been revised to include the role of available gravitational potential energy, and the relative importance of the various energy pathways has been quantified using direct numerical and large eddy simulations of convection in a three-dimensional, wide layer geometry. The energy source is the available potential energy generated by the buoyancy forcing at the boundaries. At $\mathrm{Ra} \sim 10^{7}$ small- and large-scale motions receive energy at comparable rates from the available potential energy, via buoyancy fluxes, and there is little exchange of kinetic energy between the scales. Relatively coherent flow structures result. At $\mathrm{Ra}>10^{10}$ a large proportion of the APE goes into large-scale motions, leading to the generation of small-scale turbulence by shear production. This occurs throughout the domain, and is particularly dominant inside the boundary layer. A stronger large-scale flow and a relatively less coherent structure result. The irreversible mixing, which must balance the rate of APE generation by boundary buoyancy forcing, is largely confined to the thermal boundary layer. This contribution from the boundary layer increases with increasing Rayleigh number. Indeed, the relative values of power transferred through the various energy pathways all approach asymptotes (of 0 or 1) with increasing $\mathrm{Ra}$, a result that leaves open the possibility that the conclusions drawn here may continue to apply at much larger Rayleigh numbers.

Numerical computations were carried out using the Australian National Computational Infrastructure, ANU. This work was supported by Australian Research Council Grant No. DP120102744, and G. O.H. was supported by ARC Future Fellowship FT100100869.

*Corresponding author. Bishakhdatta.Gayen@anu.edu.au

[1] Q. Zhou, C. Sun, and K.-Q. Xia, Phys. Rev. Lett. 98, 074501 (2007).

[2] G. Ahlers, S. Grossmann, and D. Lohse, Rev. Mod. Phys. 81, 503 (2009).

[3] D. Lohse and K.-Q. Xia, Annu. Rev. Fluid Mech. 42, 335 (2010).

[4] R. J. A. M. Stevens, D. Lohse, and R. Verzicco, J. Fluid Mech. 688, 31 (2011).

[5] G. Ahlers, E. Bodenschatz, D. Funfschilling, S. Grossmann, X. He, D. Lohse, R. J. A. M. Stevens, and R. Verzicco, Phys. Rev. Lett. 109, 114501 (2012).

[6] Q. Zhou and K.-Q. Xia, J. Fluid Mech. 721, 199 (2013).
[7] J. W. Deardorff and G. E. Willis, J. Fluid Mech. 28, 675 (1967).

[8] E. D. Siggia, Annu. Rev. Fluid Mech. 26, 137 (1994).

[9] E. N. Lorenz, Tellus 7, 157 (1995).

[10] K. B. Winters, P. N. Lombard, J. J. Riley, and E. A. D’Asaro, J. Fluid Mech. 289, 115 (1995).

[11] W. R. Peltier and C. P. Caulfield, Annu. Rev. Fluid Mech. 35, 135 (2003).

[12] G. O. Hughes, A. McC. Hogg, and R. W. Griffiths, J. Phys. Oceanogr. 39, 3130 (2009).

[13] B. Gayen, R. W. Griffiths, G. O. Hughes, and J. A. Saenz, J. Fluid Mech. 716, R10 (2013).

[14] G. O. Hughes, B. Gayen, and R. W. Griffiths, J. Fluid Mech. 729, R3 (2013).

[15] R. J. A. M. Stevens, R. Verzicco, and D. Lohse, J. Fluid Mech. 643, 495 (2010).

[16] S. J. Kimmel and J. A. Domaradzki, Phys. Fluids 12, 169 (2000).

[17] S. H. Peng, K. Hanjalic, and L. Davidson, J. Turbul. 7, N66 (2006).

[18] D. Chung and D. I. Pullin, J. Fluid Mech. 643, 279 (2010).

[19] O. Shishkina and C. Wagner, J. Fluid Mech. 599, 383 (2008).

[20] V. Armenio and S. Sarkar, J. Fluid Mech. 459, 1 (2002).

[21] B. Gayen, S. Sarkar, and J. R. Taylor, J. Fluid Mech. 643, 233 (2010).

[22] L. P. Kadanoff, Phys. Today 54, No. 834 (2001).

[23] J. J. Niemela, L. Skrbek, K. R. Sreenivasan, and R. J. Donnelly, Nature (London) 404, 837 (2000).

[24] J. J. Niemela, L. Skrbek, K. R. Sreenivasan, and R. J. Donnelly, J. Fluid Mech. 449, 169 (2001).

[25] R. H. Kraichnan, Phys. Fluids 5, 1374 (1962).

[26] D. Lohse and F. Toschi, Phys. Rev. Lett. 90, 034502 (2003).

[27] R. Verzicco and R. Camussi, J. Fluid Mech. 477, 19 (2003).

[28] X. Chavanne, F. Chillà, B. Chabaud, B. Castaing, and B. Hébral, Phys. Fluids 13, 1300 (2001).

[29] P.-E. Roche, F. Gauthier, R. Kaiser, and J. Salort, New J. Phys. 12, 085014 (2010).

[30] R. Tailleux, J. Fluid Mech. 638, 339 (2009).

[31] R. M. Kerr, Phys. Rev. Lett. 87, 244502 (2001).

[32] X.-D. Shang, X.-L. Qiu, P. Tong, and K.-Q. Xia, Phys. Rev. Lett. 90, 074501 (2003).

[33] S. Grossmann and D. Lohse, Phys. Rev. E 66, 016305 (2002).

[34] R. Ni and S.-D. Huang, and K.-Q. Xia, Phys. Rev. Lett. 107, 174503 (2011).

[35] B. Puthenveettil and J. H. Arakeri, J. Fluid Mech. 542, 217 (2005).

[36] S. B. Dalziel, M. D. Patterson, C.P. Caulfield, and I. A. Coomaraswamy, Phys. Fluids 20, 065106 (2008). 